\title{
Antimicrobial Properties of a Traditionally and Specially Prepared Oil Complex: NigellaSativa Seed Oil, Rosemary Oil, and Olive Oil
}

\author{
Ahmet Ozbek $^{1, a, *}$, Tayfur Demiray ${ }^{2, b}$, Elif Koptaget $^{3, c}$, Ozlem Kucuk $^{3, d}$, Leyla Demir $^{3, e}$ \\ ${ }^{1}$ Department of Medical Microbiology, Faculty of Medicine, Sakarya University, 54290 Sakarya, Turkey \\ ${ }^{2}$ Clinical Microbiology Laboratory, Sakarya University Education and Research Hospital, 54290 Sakarya, Turkey \\ ${ }^{3}$ Institute of Health Science, Sakarya University, 54290 Sakarya, Turkey
}

*Corresponding author

\begin{tabular}{l|l}
\hline A R T I C L E I N F O & A B S T R A C T \\
\hline Research Article & $\begin{array}{l}\text { Herbal medicine also called botanical or phyto-medicine refers to using a plant's seeds, berries, roots, } \\
\text { leaves, bark, or flowers for medicinal purposes. It is becoming more main stream as improvements } \\
\text { in analysis and quality control along with advances in clinical research show the value of herbal } \\
\text { medicine in the treating and preventing disease. This study was focused on a traditionally prepared } \\
\text { and used a product consist of an oil complex (olive oil, Nigella sativa seed oil, rosemary oil); which } \\
\text { were researched antimicrobial effect of the product alone, and each oil by disc diffusion methods. } \\
\text { Gram-positive and -negative bacteria and yeasts were employed in the susceptibility tests. The } \\
\text { antibiotics of imipenem and vancomycin were exploited as positive controls. While there wasn't } \\
\text { effect on Gram-negative bacteria, strong antimicrobial effects were observed on Gram-positive } \\
\text { Accepted : 07/05/2019 } \\
\text { bacteria and yeasts. Nigella sativa seed oil was the most effective. There wasn't antagonistic and/or } \\
\text { synergetic effect for the oil complex product. Although essential oils may be assigned the same } \\
\text { name, they may differ widely as antimicrobial agents due to the extraction method used, which may } \\
\text { explain the distinctive antimicrobial results reported in different studies. This was the first study to } \\
\text { investigate the antibacterial and antifungal effects of this herbal medicine. }\end{array}$ \\
$\begin{array}{l}\text { Keywords: } \\
\text { Rosemary oil } \\
\text { Olive oil } \\
\text { Oil complex } \\
\text { Antimicrobial effect }\end{array}$ & \begin{tabular}{l} 
andiva \\
\hline
\end{tabular}
\end{tabular}

\begin{abstract}
(iD https://orcid.org/0000-0001-8938-6533 (iD) https://orcid.org/0000-0001-7455-0943

b@tayfurdemiray@gmail.com

ozlem.kucukl@org.sakarya.edu.tr

(iD https://orcid.org/0000-0003-1161-4684

(i) https://orcid.org/0000-0002-1400-7911

\section{Introduction}

Plants carry out important biological functions such as self-protection against attack from bacteria, fungi, insects, and herbivorous mammals. Many such functions are mediated via a wide variety of chemical compounds, many of which have beneficial effects on human health when consumed and can be used effectively to treat human diseases. The use of plants as therapeutic agents in medicine predates written human history. Ethnobotany, the study of traditional human uses of plants, can be informative for the discovery of future medicines Elumalai and Chinna (2012), Fabricant and Farnsworth (2001), Lai and Roy (2004), and Gupta et al. (2018) indicated these issues in their studies. Treating diseases with herbs is common in non-industrialised countries, where herbs can be purchased more affordably than expensive modern pharmaceuticals (Elumalai and Chinna, 2012). With recent scientific evidence for the effectiveness of herbal medicines, applications of herbal medicines have become increasingly more common. In addition to searching for new medicinal herbs, many scientists are exploring new uses for existing traditional herbs. In this respect, many researchers have focused on a traditionally used herbal medicine product. With this study, we have focused on a product which consists of a complex of essential oils prepared traditionally and used especially in Middle and Eastern Anatolia for centuries. This traditionally used product composed of three main oil derived from olive oil, Nigella sativa seed oil, and rosemary oil. This complex of essential oils is traditionally used as a nasal dropin patient with sinusitis, one drop in each nostril for 1-3 days. We investigated the antibacterial and antifungal effects of this traditional herbal medicine. Despite the information available regarding the three essential oils individually, we have found no information on the antibacterial activity of this complex as a whole. This study is the first to investigate the antibacterial and antifungal effects of this oil complex. 


\section{Materials and Methods}

\section{Essential oils}

The product and three essential oils were obtained from NBV İlaç, Doğal Ted. Ürün Koz. Gıda ve Mad. Paz. San. Tic. LTD. ŞTİ. (Konya, Turkey), producer of the plant essential oils and the herbal medicine. The quality of the oils was $>98.5 \%$. These were dissolved in dimethylsulfoxide (DMSO) at a 1:1 ratio.

\section{Test organisms}

We tested Gram-negative and Gram-positive standard strains, fungi, and clinical isolates, which were identified by the conventional and automated identification system, Vitek 2. The Gram-negative standard strains were Escherichia coli (ATCC 25922), Klebsiellae pneumoniae (ATCC 15380), Pseudomonas aeruginosa (ATCC 10145), and Proteus vulgaris (ATCC 7002); the Gram-positive standard strains were Staphylococcus aureus (ATCC 29213) and Enterococcus faecalis (ATCC 29212); and the standard fungi were Candida albicans (ATCC 10231) and Candida tropicalis (ATCC 13803). All clinical isolates were obtained from a culture stock library at the Clinic Diagnostic Laboratory of Medical Microbiology Department of our University Hospital.

\section{Antimicrobial assay}

To screen for the antibacterial and antifungal effects of the oil complex product and three essential oils, the disc diffusion method was employed as described previously by Agaoglu et al. (2005), Prabuseenivasan et al. (2006), Stefanovic et al. (2009), and Erturk et al. (2010), with little modification. Screening was performed using a $16-18 \mathrm{~h}$ culture at $36 \pm 1^{\circ} \mathrm{C}$ in $10 \mathrm{~mL}$ Mueller-Hinton broth. The cultures were adjusted to approximately $10^{5} \mathrm{CFU} / \mathrm{mL}$ with sterile saline solution; $500 \mu \mathrm{L}$ suspensions were spread over Mueller-Hinton agar plates $(94 \mathrm{~mm} \times 16 \mathrm{~mm})($ SigmaAldrich) using a sterile cotton swab to ensure uniform microbial growth. Mueller-Hinton agar plates with 5\% sheep blood in media were used only with the streptococcus species, $S$. pyogenes and $S$. pneumoniae. Two Mueller-Hinton agar plates were employed for each of the bacterial and fungal samples. Under aseptic conditions, we used five blank discs (CT 998 B; Oxoid) and one positive control disc (30 mg vancomycin; BD$\mathrm{BBL}$ ) for Gram-positive bacteria, and $10 \mathrm{mg}$ imipenem (BD-BBL) for Gram-negative bacteria. Blank discs were placed on the agar surface, arranged as follows: three discs for the three essential oils (olive oil, Nigella sativa seed oil, and rosemary oil), one disc for vehicle control (negative control), and one disc for the essential oil complex product. The three essential oils were examined on one MuellerHinton agar plate, and the positive and negative controls and the essential oil complex product were examined on the other Mueller-Hinton agar plate. After placing the discs, the agar plates with media were incubated at room temperature for $5 \mathrm{~min}$ to attach the discs on the media surface. Each essential oil $(20 \mu \mathrm{L})$ was pipetted onto the related discs, and then the plates were placed in an incubator at $36 \pm 1{ }^{\circ} \mathrm{C}$ for $18 \mathrm{~h}$. These were incubated coverside up for $1 \mathrm{~h}$ to allow diffusion of the oil, and then they were inverted for the remainder of the incubation.

\section{Results and Discussion}

The antibacterial activities of three essential oils and the oil complex product were tested against 13 bacterial species and 3 fungi species (Table 1).

Thirteen different bacteria genera (including 52 strains) and two different yeast genera (including 5 strains) were included in the evaluation. A sample was scored as positive when the zone of inhibition of the sample was greater than or equal to that of the positive control. No antibacterial effect was observed against Gram-negative bacteria; however, all Gram-positive bacteria and yeast showed sensitivity. The results showed that the main antimicrobial effect originated from Nigella sativa seed oil. Although each of the oils in the mixture displayed antimicrobial effects separately, the oil complex showed no synergetic effect. All vehicle controls (negative controls) showed no inhibition of growth.

Traditional medicine or complementary medicine has a long history of use in health maintenance, disease prevention, and treatment of chronic disease. In this respect, traditional medicine has great potential to improve health and wellness; however, it requires particular attention to avoid misunderstandings and inappropriate use that could be harmful to individuals. There is a growing trend in which people, with or without health problems, are taking a more proactive approach to their own health and seeking out different forms of self-care. Many of these individuals have an assumption that natural means safe. Almost one quarter of all modern therapeutic drugs originate from natural products, many of which were used first in a traditional medicine product. Therefore, traditional medicine is a resource for primary health care but also for innovation and discovery. Working together, traditional and modern medicine systems might produce beneficial harmony, taking advantage of the best features of each system and compensating for certain weaknesses of the other as mentioned by Basmacioglu-Malayoglu et al. (2011), WHO traditional Strategy 2014-2023 (2013), Barnes (2003), Hassan et al. (2009), and Zhang (2018). This study examined the utility of a traditional complex in a modern medicine system by analysing the product's antimicrobial and antifungal effects.

Although the essential oils and the herbal medicine showed no antibacterial effects on Gram-negative bacteria, we found strong effects on Gram-positive bacteria and yeasts in the micro-organisms examined. The antibacterial effects on Enterococcus faecalis, Enterococcus feacium, and Enterococcus gallinarum originated from Nigella sativa seed oil. The antibacterial effects on Streptococcus pyogenes and Streptococcus pneumonia originated not only from the Nigella sativa seed oil alone, but also from olive oil and rosemary oil. These two essential oils alone appeared weaker than Nigella sativa seed oil, as the effect of olive oil showed a borderline effect that was less than that of the positive control. The antibacterial effects on the staphylococcus species $S$. aureus, $S$. haemolyticus, and $S$. epidermidis were as follows: olive oil had no effect on $S$. aureus or $S$. haemolyticus but had a slight effect against S.epidermidis. Rosemary oil had a weaker effect than Nigella sativa seed oil but a greater one than olive oil. Olive oil showed no effect against the yeasts $C$. albicans, C. tropicalis, and C. neoformans, whereas the other oils did, with Nigella sativa seed oil being the most effective. 
Table 1 Antimicrobial activities of the essential oils and the oil complex product

\begin{tabular}{|c|c|c|c|c|}
\hline \multirow[t]{2}{*}{ Microorganism } & \multicolumn{4}{|c|}{$\begin{array}{l}\text { Antimicrobial activity } \\
\text { (inhibition zone, } \mathrm{mm} \text { ) }\end{array}$} \\
\hline & A & $\mathrm{B}$ & $\mathrm{C}$ & OCP \\
\hline \multicolumn{5}{|l|}{ Gram-negative bacteria } \\
\hline Escherichia coli ATCC 25922 and four clinical isolates & none & none & $7.0( \pm 1)$ & $5.0( \pm 1)$ \\
\hline $\begin{array}{l}\text { Pseudomonas aeruginosa ATCC 27833, ATCC } 10145 \\
\text { and four clinical isolates }\end{array}$ & none & none & none & none \\
\hline Acinetobacter baumannii: four clinical isolates & none & none & none & none \\
\hline Klebsiella pneumonia: four clinical isolates & none & none & none & none \\
\hline Proteus vulgaris (ATCC 7002) and four clinical isolates & none & none & none & none \\
\hline \multicolumn{5}{|l|}{ Gram-positive bacteria } \\
\hline $\begin{array}{l}\text { Enterococcus faecalis (ATCC 29212) and four clinical } \\
\text { isolates }\end{array}$ & none & $19.3( \pm 0.2)$ & none & $18.5( \pm 0.3)$ \\
\hline Enterococcus faecium four clinical isolates (VRE) & none & $20.1( \pm 0.2)$ & none & $19.2( \pm 0.2)$ \\
\hline Enterococcus gallinarum four clinical isolates (VRE) & none & $20.4( \pm 0.1)$ & none & $19.3( \pm 0.1)$ \\
\hline Streptococcus pyogenes four clinical isolates & $17.0( \pm 0.1)$ & $46.8( \pm 0.2)$ & $22.0( \pm 0.1)$ & $45.3( \pm 0.3)$ \\
\hline Streptococcus pneumonia four clinical isolates & $17.0( \pm 0.1)$ & $47.6( \pm 0.2)$ & $22.0( \pm 0.1)$ & $46.0( \pm 0.3)$ \\
\hline $\begin{array}{l}\text { Staphylococcus aureus ATCC } 29213 \text { and four clinical } \\
\text { isolates }\end{array}$ & none & $58.0( \pm 0.3)$ & $12.0( \pm 0.1)$ & $52.0( \pm 0.3)$ \\
\hline Staphylococcus haemolyticus four clinical isolates & none & $59.7( \pm 0.2)$ & $12.4( \pm 0.2)$ & $55.2( \pm 0.3)$ \\
\hline Staphylococcus epidermidis four clinical isolates & $12.0( \pm 0.2)$ & $61.8( \pm 0.2)$ & $23.2( \pm 0.1)$ & $57.3( \pm 0.3)$ \\
\hline \multicolumn{5}{|l|}{ Yeast } \\
\hline Candida albicans ATCC 10231 and four clinical isolates & none & $42.1( \pm 0.2)$ & $15.3( \pm 0.1)$ & $36.3( \pm 0.1)$ \\
\hline $\begin{array}{l}\text { Candida tropicalis (ATCC 13803) and four clinical } \\
\text { isolates }\end{array}$ & none & $42.0( \pm 0.3)$ & $16.2( \pm 0.1)$ & $38.0( \pm 0.1)$ \\
\hline Cryptococcus neoformans one clinical isolate & none & $39.1( \pm 0.1)$ & $13.1( \pm 0.2)$ & $35.3( \pm 0.1)$ \\
\hline Enterobacteriaceae & \multicolumn{4}{|c|}{ IMP $\geq 23$} \\
\hline Acinetobacter baumannii & \multicolumn{4}{|c|}{ IMP: $\geq 22$} \\
\hline Pseudomonas aeruginosa & \multicolumn{4}{|c|}{ IMP: $\geq 19$} \\
\hline Enterococcus faecalis / faecium & \multicolumn{4}{|c|}{$\mathrm{VA}^{\mathrm{I}}: \geq 17$} \\
\hline S.pyogenes, S. pneumoniae & \multicolumn{4}{|c|}{$\mathrm{VA}: \geq 17$} \\
\hline
\end{tabular}

Notes: A, olive oil; B, Nigella sativa seed oil; C, rosemary oil; OCP, the oil complex product; VRE, vancomycin-resistant enterobacteria; IMP, imipenem; VA, vancomycin; CLSI 2014 M100-S24

Viljoen et al. (2003) and Fu et al. (2007) had been indicated in their experiments; the main compounds found in rosemary essential oil are 1,8-cineole, $\alpha$-pinene, and camphor, which have antibacterial and antifungal effects. Camphor and 1,8-cineole are reported to have antimicrobial effects, and camphor is reported to have antiseptic and decongestive effects (Viljoen et al., 2003). The topical use of camphor for fibrosis has also reported (Hasan et al., 2013). Viljoen et al. (2003), Khan (1999), Akgul et al. (1989), Carson and Riley (1995) and Shunying et al. (2005) indicated in their researches, cineole has antibacterial effects against Gram-positive samples but not against $P$. aeruginosa (Pattnaik et al., 1997). Rosemary oil has strong antibacterial effects, with consistent inhibition against Gram-negative and Gram-positive bacteria (Prabuseenivasan et al., 2006). In the present study, rosemary oil had antibacterial effects against $S$. pyogenes, S. pneumonia, S. aureus, S. haemoliticus, and $S$. epidermidis. It also had antifungal effects against $C$. albicans, C. tropcalis, and C. neoformans. Previously, cineole was found to have antifungal activity, especially against $C$. albicans (Viljoen et al., 2003).

Nigella sativa seed oil has many advantageous medical properties, and is universally called a panacea (Khan 1999). Notably, of the three oils, it showed the most effective antibacterial and antifungal in the present study, and had almost the same antibacterial and antifungal effects as the oil complex product, which included all three essential oils together with some supplements. Therefore, the concentration of Nigella sativa seed oil was reduced in the complex, but its effects alone nearly matched that of the complex, implying that it accounted for most of the overall effect. Previous studies managed by Hanafy and Hatem (1991), Morsi (2000), and Salman et al., (2008) that examined the antibacterial and antifungal effects of Nigella sativa seed oil showed different results against Gramnegative bacteria compared to our study. However, Hasan et al., (2013) detected lower antibacterial activity against Gram-negative than against Gram-positive bacteria, similar to our results. Medina et al., (2006), Medina et al., (2007), and Shan et al., (2013) indicated in their study that olive oil and olive leaf extracts have been used in traditional medicine for centuries because of their antimicrobial activity, which originates from phenolic compounds. In the present study, we observed a borderline antibacterial effect on S. pyogenes and S. pneumonia only.

An important characteristic of essential oils and their components is their hydrophobicity, which enables them to partition in the lipid layers of the bacterial cell membrane and mitochondria, disturbing the structures and rendering them more permeable. Thus, leakage of the cell contents can occur. Although cells can tolerate a certain amount of leakage without loss of viability, extensive loss of cell contents or exit of critical molecules and ions leads to cell death (Burt, 2004). Essential oils or aromatic plant essences are volatile, and fragrant substances that have oily consistencies are frequently produced by plants. Such substances can be in liquid form at room temperature, 
although several are solid or resinous. Some substances show different colours ranging from pale yellow to emerald green and from blue to dark brownish-red. These substances can be synthesised by a variety of plant organs, i.e., buds, flowers, leaves, stems, twigs, seeds, fruits, roots, wood, and bark, and are stored in secretory cells, cavities, canals, epidermic cells, and glandular trichomes. Essential oils from different parts of an aromatic plant can be extracted by various techniques, including water or steam distillation, solvent extraction, expression under pressure, and supercritical and subcritical water extractions (Bassolel and Juliani, 2012). Therefore, although essential oils may be assigned the same name, they may differ widely as antimicrobial agents due to the extraction method used. This difference in extraction method may explain the distinctive antimicrobial results reported in different studies.

In conclusion, the oil complex had strong antimicrobial effects on standard and clinically isolated microorganisms, but no synergistic effect was observed, and Nigella sativa seed oil was mainly responsible for the antibacterial activity of the complex. In fact, Nigella sativa seed oil alone slightly outperformed the complex. This could be explained by the reduced amount of Nigella sativa seed oil in the product.

This is the first study to examine this oil complex product, which is still used in traditional medicine. In an ideal world, traditional medicine would be an option and choice, offered by a well-functioning, people-centred health system that balances curative services with preventive care. The challenge is to appropriately include traditional medicine in an integrated health system, to help all practitioners understand its unique and valuable contribution, and to educate consumers about what it can and cannot do. In other words, this rich resource and cultural heritage must be modernised and evaluated with respect to accepted Western practises. Further studies are needed to understand the medicinal properties of this composition.

\section{References}

Agaoglu S, Dostbil N, Alemdar S. 2005. Antimicrobial effect of seed extract of cardamom (Elettaria cardamomum Maton). YYU Vet Fak Derg, 16(2): 99-101.

Akgul A, Kivanc M, Bayrak A. 1989. Chemical Composition and Antimicrobial Effect of Turkish Laurel Leaf Oil. J Essent Oil Res, 1(6): 277-280.

Barnes J. 2003. Quality efficacy and safety of complementary medicines: Fashions, facts and future. Part II: Efficacy and safety. Br J Clin Pharmacol, 55(4): 331-340.

Basmacioglu-Malayoglu H, Ozdemir P, Hames-Kocabas EE. 2011. Chemical compositions and antibacterial activity of the essential oils from some plant species. Ege Univ Ziraat Fak Derg, 48(1): 11-18.

BassoleI H, Juliani HR. 2012. Essential oils in combination and their antimicrobial properties. Molecules, 17(4): 3989-4006.

Burt S. 2004. Essential oil: their antibacterial properties and potential applications in foods-a review. Int J Food Microbiol, 94:223-253.

Carson CF, Riley TV. 1995. Antimicrobial activity of the major components of the essential oil of Melaleuca alternifolia. J of App Bacteriol, 78(3): 264-269.

Elumalai A, Chinna Eswariah M. 2012. Herbalism. Inter J of Phytotherapy, 2(2): 96-105.
Erturk R, Celik C, Kaygusuz R, Aydın H. 2010. Antimicrobial activities of commercial essential oils of thyme and mint. Cumhuriyet Med J, 32: 281-286.

Fabricant DS, Farnsworth NR. 2001. The value of plants used in traditional medicine for drug discovery. Environ Health Perspect, 109(Suppl 1): 69-75.

Fu Y, Zu Y, Chen L, Shi X, Wang Z, Sun S, Efferth T. 2007. Antimicrobial activity of clove and rosemary essential oils alone and in combination. Phytother Res, 21(10): 989-994.

Gupta S, Jain R, Kachhwaha S, Kothari SL. 2018. Nutritional and medicinal applications of Moringa oleifera Lam.--Review of current status and future possibilities. Journal of Herbal Medicine, 11: 1-11.

Hanafy MS, Hatem ME. 1991. Studies on the antimicrobial activity of nigella sativa

Hasan NA, Nawahwi MZ, Malek HA. 2013. Antimicrobial activity of Nigella sativa seed extract. Sains Malaysiana, 42(2):143-147.

Hasan SS, Ahmet SI, Irfan Bukhari NI, Loon WC. 2009. Use of complementary and alternative medicine among patients chronic diseases at outpatient clinics. Complement Ther Clin Pract, 15(3): 152-157.

Khan MA. 1999. Chemical composition and medicinal properties of nigella sativa Linn. Inflammopharmacology, 7(1): 15-35.

Lai PK, Roy J. 2004. Antimicrobial and chemopreventive properties of herbs and spices. Curr Med Chem, 11(11): 1451-1460.

Medina E, de Castro A, Romero C, Brenes M. 2006. Comparison of the concentrations of phenolic compounds in olive oils and other plant oils: Correlation with antimicrobial activity. J Agric Food Chem, 54(14): 4954-4961.

Medina E, Romero C, BrenesM, de Castro A. 2007. Antimicrobial activity of olive oil, vinegar, and various beverages against foodborne pathogens. J Food Prot, 70(5): 1194-1199.

Morsi NM. 2000. Antimicrobial effect of crude extracts of Nigella sativa on multiple antibiotics-resistant bacteria. Acta Microbiol Pol, 49(1): 63-74.

Pattnaik S, Subramanyam VR, Bapaji M, Kole CR. 1997. Antibacterial and antifungal activity of aromatic constituents of essential oils. Microbios, 89(358): 39-46.

Prabuseenivasan S, Jayakumar, M, Ignacimuthu S. 2006. In vitro antibacterial activity of some plant essential oils. BMC Complement Altern Med, 6(39) doi:10.1186/1472-6882-6-39.

Salman MT, Khan RA, Shukla I. 2008. Antimicrobial activity of Nigella sativa Linn. seed oil against multi-drug resistant bacteria from clinical isolates. Natural Product Radiance, $7(1): 10-14$.

seed (black cumin). J Ethnopharmacol. 34(2-3): 275-278.

Shah QA, Bibi F, Shah AH. 2013. Anti-microbial effects of olive oil and vinegar against salmonella and Escherichia coli. PJST, 14(2): 479-486.

Shunying Z, Yang Y, Huaidong Y, Yue Y, Guolin Z. 2005. Chemical composition and antimicrobial activity of the essential oils of Chrysanthemum indicum. J Ethnopharmacol, 96(1-2): 151-158.

Stefanovic O, Comic L, StefanovicD, Solujic-Sukdolak S. 2009. Antibacterial activity of Aegopodium podagraria L. extracts and interaction between extracts and antibiotics. Turk J Biol, 33:145-150.

Viljoen A, van Vuuren S, Ernst E, Klepser M, Demirci B, Baser H, Wyk BEV. 2003. Osmitopsisasteriscoides (Asteraceae)the antimicrobial activity and essential oil composition of a Cape-Dutch remedy. J Ethnopharmacol, 88(2-3): 137-143.

WHO traditional Strategy 2014-2023. 2013. World Health Organisation, [Online] Available: (https://www.who.int/ medicines/publications/traditional/trm_strategy14_23/en/)

Zhang Q. 2018. Global situation and WHO strategy on traditional medicine. Traditional Medicine and Modern Medicine, 1(1): 11-13. 